\title{
Supramolecular Polymer-Molecule Complexes as Gain Media for Ultraviolet Lasers
}

\author{
Jin-Yi Lin, ${ }^{\dagger,}, \|$ Gang-Yi Zhu, ${ }^{\S}$ Bin Liu, ${ }^{\ddagger}$ Meng-Na Yu, ${ }^{\ddagger}$ Xu-Hua Wang,,$^{\prime}$ Long Wang, ${ }^{\ddagger}$ Wen-Sai Zhu, ${ }^{\dagger}$ \\ Ling-Hai Xie, ${ }^{*, \ddagger}$ Chun-Xiang Xu, ${ }^{*, \S}$ Jian-Pu Wang, ${ }^{\dagger}$ Paul N. Stavrinou, ${ }^{\| \wedge}$ Donal D. C. Bradley, ${ }^{*, \|, \&}$ Wei \\ Huang*十,‡
}

${ }^{\dagger}$ Key Laboratory of Flexible Electronics (KLOFE) \& Institute of Advanced Materials (IAM), Jiangsu National Synergetic Innovation Center for Advanced Materials (SICAM), Nanjing Tech University (NanjingTech), 30 South Puzhu Road, Nanjing, China

${ }^{\ddagger}$ Key Laboratory for Organic Electronics and Information Displays \& Institute of Advanced Materials (IAM), Jiangsu National Synergetic Innovation Center for Advanced Materials (SICAM), Nanjing University of Posts \& Telecommunications, 9 Wenyuan Road, Nanjing, China

${ }$ Centre for Plastic Electronics, Department of Physics, The Blackett Laboratory, Imperial College London, Prince Consort Road, London SW7 2AZ, UK

$\wedge$ Department of Engineering Science, University of Oxford, Parks Road, Oxford OX1 3PJ, UK

${ }^{\&}$ Departments of Engineering Science and Physics and Division of Mathematical, Physical and Life Sciences, University of Oxford, 9 Parks Road, Oxford OX1 3PD, UK

§State Key Laboratory of Bioelectronics, School of Biological Science and Medical Engineering, Southeast University, Nanjing, China

\begin{abstract}
A novel supramolecular system comprising a complex of 9,9'-diphenyl-9H,9'H-2,2'-bifluorene-9,9'-diol (DPFOH) with poly(methylmethacrylate) (PMMA) is presented as an attractive system for optical gain in the ultraviolet. The analogue compound 9,9'-diphenyl-9H,9'H-2,2'-bifluorene (DPFO8) without -OH substituent was synthesized alongside DPFOH to confirm the importance of its chemical structure to the thin film microstructure. A hydrogen-bonding interaction allows the molecule such as DPFOH and a combination of DPFOH and PMMA to have an excellent solution-processed high quality coating film. In stark contrast to DPFO8 system, we find that the addition of $1 \mathrm{wt} \%$ DPFOH to PMMA leads to spontaneous formation of a supramolecular complex via hydrogen bonding interactions, giving rise to a homogenous film with relatively high photoluminescence quantum efficiency $\sim 38( \pm 5) \%$. The demonstration of ultraviolet laser action with peak wavelength emission at $385 \mathrm{~nm}$ provided further evidence of the high optical quality of the DPFOH/PMMA supramolecular complex films. DPFOH-PMMA supramolecular complex has great potential for use in low-cost solution processed optoelectronic devices.
\end{abstract}

Solution-processed organic optoelectronic devices have long attracted worldwide research and commercial interest, mainly due to the associated low manufacturing costs and the potential for large area production using high throughput printing and other coating techniques. ${ }^{1-4}$ In addition to the optoelectronic properties, molecular design plays a key role in the processability and thermal stability of organic materials. Adopting a programmable supramolecular approach can bring further opportunities for solution processed conjugated systems. ${ }^{5-7} \mathrm{~A}$ supramolecular system or "supermolecule" is a well-defined complex of molecules held together by non-covalent bonds. The engineering of these assemblies offers a key to new possibilities for optoelectronic materials and devices. Supramolecular polymeric materials comprising an aromatic organic molecule can be classified into three categories ${ }^{8}$ (I) Supramolecular polymeric semiconductor (SPS). ${ }^{9-15}$ (II) Conjugated supramolecular polymer (CSP) 7, 8, 16 and (III) Supramolecular doped polymer (SDP). ${ }^{8,}{ }^{17}$ Herein, our interest is to develop a supramolecular system based on the SDP approach. Conjugated dopant unit of the supramolecule are grafted onto a nonconjugated polymer chain via hydrogen bond interactions. ${ }^{8}$
We report a new DPFOH-PMMA SDP supramolecular system and compare it with films of PMMA doped with the nonhydrogen-bonding DPFO8, describing a range of structural and optoelectronic properties. PMMA is widely used as a polymer optical fiber (POF) material; largely due to its high transparency, flexibility, and low cost processing. ${ }^{18,19}$ For the SDP dopant we used a newly synthesized fluorescent molecule, namely 9,9'-diphenyl-9H,9'H-2,2'-bifluorene-9,9'-diol (DPFOH, Figure 1(a)). Fluorophore-doped PMMA SDP systems of this type could be promising candidates with which to realize polymer optical fibre amplifiers (POFAs), provided, of course, that they exhibit suitably high-quality optical properties, especially in respect of optical gain. ${ }^{18,20}$ Among the challenges for such multi-component blended material systems is to obtain low scattering losses. This is dependent on fabricating uniform films with homogeneous microstructures. A key requirement is thus to avoid phase separation between the components and the introduction of supramolecular interactions between them can be helpful in this regard. ${ }^{21,22}$ In the present work, we take full advantage of the SDP approach to arrive at a gain medium for use in ultraviolet lasers. 

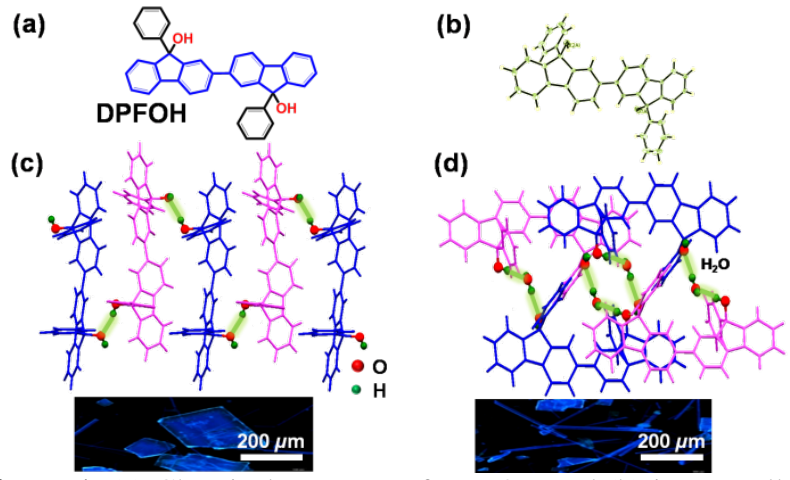

Figure 1. (a) Chemical structure of DPFOH and (b) its crystallographic structure. Two different molecular packing modes for $\mathrm{DPFOH}$ single crystal, without (c) and with (d) the incorporation of $\mathrm{H}_{2} \mathrm{O}$. The green line signifies the hydrogen-bonds. Insets show the fluorescence microscope (FLM) images of single crystals.

DPFOH was synthesized via a typical Suzuki cross-coupling reaction between 9-phenyl-fluorenolboronic ester and 2bromo-substituted 9-phenyl-fluorenol (Scheme S1 and Figure S1). To compare and contrast the dependence of film forming properties on supramolecular interactions, 9,9'-diphenyl9H,9'H-2,2'-bifluorene (DPFO8), without -OH substituents was also synthesized (Figure S2) ${ }^{9,23}$ Differential scanning calorimetry (DSC) and thermo gravimetric analysis (TGA) were performed to evaluate the thermal properties of DPFOH. Results from both measurements suggest that $\mathrm{DPFOH}$ exhibits outstanding thermal stability (Figures S3 and S4). A TGA decomposition temperature $\left(\mathrm{T}_{\mathrm{d}}\right)$ around $330{ }^{\circ} \mathrm{C}$ is found for $5 \%$ weight loss (Figure S3). An excellent microstructural stability is also inferred, with a crystallization exotherm appearing at $\mathrm{T}_{\mathrm{C}}=220^{\circ} \mathrm{C}$ in DSC (Figure S4) but no other distinct thermal transitions across the temperature range 30 to $230{ }^{\circ} \mathrm{C}$. Single crystal structures provide an effective means to screen intermolecular hydrogen-bonding interactions in the solution, crystalline and solid states. Single crystals of DPFOH were grown from a 3:1 volume ratio of toluene: isopropyl alcohol mixed solution. Crystallographic analysis, from single-crystal $\mathrm{X}$-ray diffraction measurements, suggested a torsion angle of $10.78^{\circ}$ between adjacent fluorene moieties (Figure 1(b-d)). As shown in Figure 1(c), from the crystallographic structure of DPFOH flake single crystal, strong supramolecular hydrogen bonding interactions between DPFOH molecules, with a characteristic distance of $2.043 \AA$ suggests the formation of a DPFOH-based CSP. The incorporation of hydrogen-bonding and $\pi-\pi$ stacking interactions promotes DPFOH lamellar crystal formation (Figure 1(c), inset). Interestingly, in the presence of $\mathrm{H}_{2} \mathrm{O}$, we obtain rod-like single crystals instead, comprising a supramolecular $\mathrm{H}_{2} \mathrm{O}$ :DPFOH complex or co-crystal (Figure 1(d)). The characteristic distance of $\mathrm{O}-\mathrm{H} \cdots \mathrm{O}$ interactions between the $\mathrm{O}-\mathrm{H}$ of DPFOH and $\mathrm{O}$ of $\mathrm{H}_{2} \mathrm{O}$ is $~ 1.940 \AA$, thereby confirming the formation of hydrogen-bonding interaction. However, the hydrogen-bonding interactions among DPFOH to $\mathrm{DPFOH}$ molecules may be disrupted via the addition of other molecules (including polymers, such as PMMA) possessing $\mathrm{O}-\mathrm{H}, \mathrm{C}=\mathrm{O}, \mathrm{H}_{3} \mathrm{CO}-\mathrm{C}=\mathrm{O}$ groups. In this regard, $\mathrm{DPFOH}$ might then exhibit single molecule photophysical behavior, with the blends acting as highly diluted solid solutions. (a)

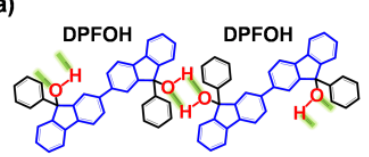

(b)
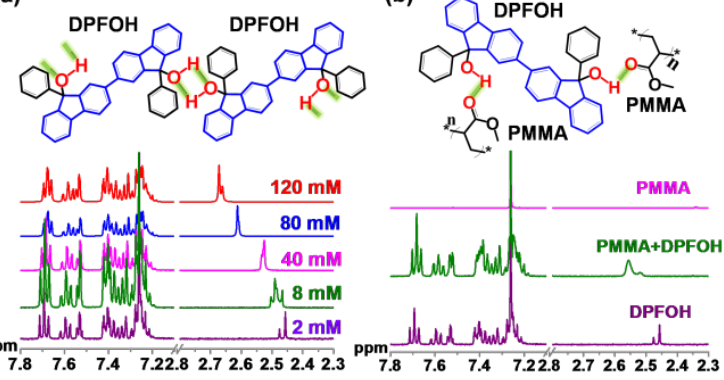

Figure 2. (a) Partial ${ }^{1} \mathrm{H}$ NMR spectra from different concentrations of DPFOH in $\mathrm{CDCl}_{3}$ solutions. (b) Comparison of ${ }^{1} \mathrm{H}$ NMR spectra for $2 \mathrm{mM}$ DPFOH and the mixture of $\mathrm{DPFOH}$ (1wt\%)/PMMA in $\mathrm{CDCl}_{3}$ solution. Green lines represent the hydrogen-bonds in SPS and SDP structures.
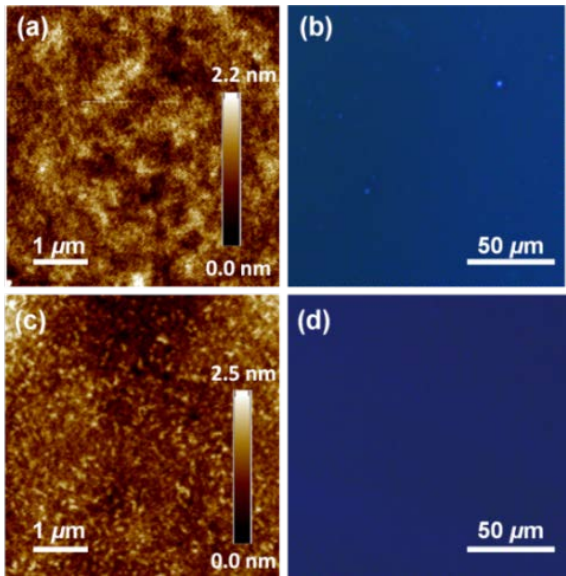

Figure 3. AFM and FLM images of a DPFOH CSP film (a, b) and a DPFOH/PMMA SDP film with the ratio of $1 \mathrm{wt} \%$ (c, d).

${ }^{1} \mathrm{H}$ NMR spectroscopy provides further insight into the DPFOH-based CSP and PMMA/DPFOH-based SDP structures. Figure 2(a) shows the concentration-dependent ${ }^{1} \mathrm{H}$ NMR spectra for $\mathrm{DPFOH}$ in deuterated chloroform $\left(\mathrm{CDCl}_{3}\right)$. At low concentration, below $2 \mathrm{mM}$, the $\mathrm{H}_{\delta}$ of the hydroxyl group is around $2.45 \mathrm{ppm}$. On increasing the DPFOH concentration up to $120 \mathrm{mM}$, however, the corresponding hydroxyl group signal shifts from 2.45 to $2.67 \mathrm{ppm}$, suggesting the formation of intermolecular hydrogen-bonding interactions between DPFOH and adjacent DPFOH molecule. This observation supports the proposed formation of a high molecular weight CSP driven by intermolecular hydrogen-bonding (Figure2(a)). The impact on thin-film formation is positive, with smooth $\mathrm{DPFOH}$ thinfilms (see Figure 3(a) and S5) routinely spin-coated from toluene solution. As shown in Figure 3(b), the uniformity of emission from the DPFOH spin-coated films, effectively confirms a high quality DPFOH films. On introducing PMMA into the $\mathrm{DPFOH}$ solution, the ${ }^{1} \mathrm{H}$ NMR signal from the-OH group down-shifts by $0.12 \mathrm{ppm}$ relative to $\mathrm{DPFOH}$ alone (Figure 2(b), $2 \mathrm{mM}$ in $\mathrm{CDCl}_{3}$ ). In addition to the DPFOH CSP films, the supramolecular interactions in the DPFOH/PMMA SDP system also support the production of high quality, homogeneous thin films (Figure 3(c), S7 and S8), and an efficient dispersion of fluorophores is also demonstrated via FLM images in (Figure 3(d) and S8). In stark contrast, DPFO8 selfassembles into irregular micro-fibers, nanosheets and crystals (Figure S5 and S6) and, consequently, does not form uniform thin films from solution. Furthermore, the spin-coated films from PPFO8 (1 wt\%)/PMMA either have small crystals or 
aggregate (Figure S7 and S8), suggesting that a phase separation happened in the film spin-coating process.

(a)

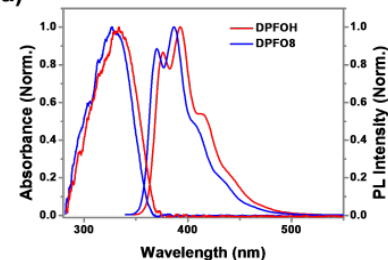

(b)

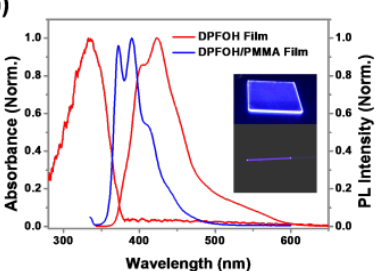

Figure 4. (a) Absorption and photoluminescence (PL) spectra forDPFO8 and DPFOH in the dilute $\left(10^{-3} \mathrm{mg} / \mathrm{mL}\right)$ solutions. (b) Absorption and PL spectra for DPFOH films ( $80 \mathrm{~nm}$ thickness) together with the PL spectrum for a DPFOH:PMMA film $(\sim 2 \mu \mathrm{m}$ thickness) coated onto an optical fiber. Inset shows photographs of a DPFOH film on a spectrosil substrate and a DPFOH:PMMA coated optical fiber, under $365 \mathrm{~nm}$ UV light excitation.

To further characterize the influence of supramolecular complex formation we turned next to measurements of sample optical properties. UV-vis absorption and photoluminescence (PL) spectra were measured for different concentration toluene solutions of DPFOH and DPFO8 (Figure 4, S9-S11). The solution absorption spectra for both DPFOH and DPFO8 have a long-wavelength peak at $\sim 325 \mathrm{~nm}$, assigned to the $\pi-\pi^{*}$ transition of the bifluorene. The DPFOH absorption is marginally red-shifted relative to that of DPFO8 but otherwise very similar in line shape. For dilute solutions, i.e. $10^{-3} \mathrm{mg} / \mathrm{mL}$, the PL spectra from both materials show clear vibronic progressions. Again, the DPFOH spectrum is slightly red shifted (by $6 \mathrm{~nm}$ ) relative to that from DPFO8 (consistent with the absorption data). The first three PL vibronic bands for DPFOH peak at $370 \mathrm{~nm}, 387 \mathrm{~nm}$ and $408 \mathrm{~nm}$. PL quantum yields ( $\Phi_{\text {PLQY }}$ ) measured for the dilute solutions above, were broadly similar and found to be $73 \%$ and $68 \%$ for the DPFOH and DPFO 8 samples, respectively. Recording the PL emission spectra as a function of solution concentration revealed some interesting differences (Figures S9 and S10). Barring the effects of strong self-absorption that are present for the 1 and $5 \mathrm{mg} / \mathrm{mL}$ solutions (Figure S9), the PL emission spectra from the DPFO8 samples were virtually identical. This implies a weak interaction among DPFO8 molecules. Conversely, over the same concentration range, the emission spectra for the DPFOH samples, show very different behavior (Figures S10, S11). As a concentration is increased, the spectral contribution at longer wavelengths noticeably rises. There is also, what appears to be, the appearance of an supramolecular aggregated emission peak at $\sim 450 \mathrm{~nm}$, (please note that an excimer may exist in the aggregation states ${ }^{26}$ ). It is clearly visible for the highest concentrations (Figure S10). Furthermore, the time-resolve PL measurements show that the decay time of emission peak at $450 \mathrm{~nm}$ is about $816 \mathrm{ps}$, longer than those for $370 \mathrm{~nm}, 387 \mathrm{~nm}$ and $408 \mathrm{~nm}$ with the decay times of 360 400 ps, supporting the formation of aggregation and the significant interactions among DPFOH molecules in the solution. The absorption spectra for DPFOH thin-film samples appear slightly broader than found for dilute solutions. They retain, however, the same absorption peak wavelength (Figure 4). In contrast, DPFO8 did not form uniform films on spin-coating from solution; a rough and discontinuous coverage of the substrates resulted and absorption spectra could not, consequently, be obtained, but PL spectra of a DPFO8 solid state in Figure S12 confirm its green emission consistent with the results in Figure S6. The corresponding DPFOH PL spectra are red-shifted by around
$30 \mathrm{~nm}$, with a clear but somewhat less-well-resolve dvibronic structure and a long-wavelength tail. The red-shift again points towards significant intermolecular interactions as does the green-band-like tail (Figure 4(b) and S12). ${ }^{24,25}$ Mindful that such interactions are potentially detrimental to the efficiency of light emission processes, we turned to the blended SDP comprising DPFOH molecules at 1 wt $\%$ concentration in PMMA. Figure 4(b) presents the PL emission spectrum (blue curve) from such a SDP film, dip-coated onto an optical fiber. It is virtually identical to that of the dilute solution (red trace in Figure 4(a)), indicating that our blended SDP films exhibit the spectral characteristics of well-dispersed DPFOH molecules. These observations are consistent with the relatively high PLQY 38\% measured for the SDP films (c f. 20\% for $\mathrm{DPFOH}$ films) and are promising for their use as gain media in lasers and/or amplifiers. ${ }^{27-28}$ (a)

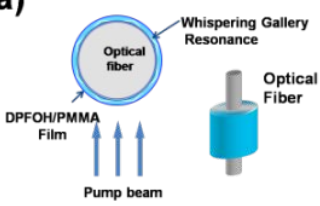

(c)

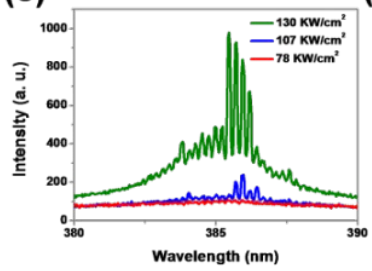

(b)

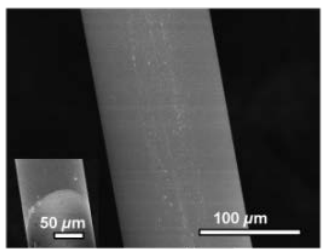

(d)

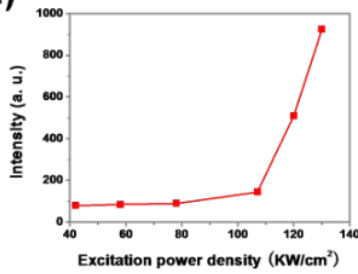

Figure 5. (a) A schematic illustration of the laser resonator structure and pumping configuration for the DPFOH:PMMA coated optical fiber. (b) SEM images of the optical fiber coated with DPFOH:PMMA. The higher resolution image shows the uniformity of the coating at its centre while the lower resolution image shows the edge region. (c) Emission spectra taken below and above threshold for whispering gallery mode lasing. (d) Spectrally integrated emission intensity as a function of excitation power.

Owing to the formation of hydrogen bonds between DPFOH and PMMA in our blended SDP films, we can effectively suppress the phase separation and/or crystallization that can otherwise occur during film formation. The result is a blended film, exhibiting good optical quality, microstructure and stability (Figure 3, S7 and S12). ${ }^{21,} 22$ As a demonstration of the utility of the DPFOH/PMMA SDP we present results for whispering gallery mode lasing using the SDP as an optical gain medium in a ring microlaser (Figure 5(a) and 5(b)). ${ }^{26,27}$ Silica optical fibers ( $125 \mu \mathrm{m}$ diameter) were dipped into a saturated chloroform solution of the SDP, resulting, following fast drying in air, in a $\sim 2 \mu \mathrm{m}$ thickness polymer over-coating, (Figure 5(b)). Optical pumping was with 6 ns pulses at 355 $\mathrm{nm}$, obtained from a Nd:YAG laser operating at $10 \mathrm{~Hz}$ repetition rate. The pump beam was focused at normal incidence onto the SDP film coated fiber (Figure 5(a)). Light emitted from the SDP films was collected by an optical fiber and coupled into an optical-multichannel analyzer (OMA) system with a charge-coupled-device (CCD) array detector (spectral resolution $0.05 \mathrm{~nm}$ ). Emission spectra, recorded at excitation intensities above and below the lasing threshold are shown in Figure 5(c). Above threshold, a series of sharp peaks, with linewidths $\delta_{\lambda} \leq 0.15 \mathrm{~nm}$, become clearly visible in the emission spectra. At a peak wavelength $\lambda_{\max }=385 \mathrm{~nm}$, the $\mathrm{Q}$ factor $(\approx$ 
$\lambda_{\max } / \delta_{\lambda}$ ) can be estimated to be around 2000. Figure 5(d) shows the integrated intensity across the 0-1 peak (380 390 $\mathrm{nm}$ ) as a function of excitation power. A clear (lasing) threshold is apparent at $\sim 103 \mathrm{~kW} / \mathrm{cm}^{2}$. The utility and success of the DPFOH:PMMA SDP can be best appreciated by comparison to DPFO8:PMMA blend films, which we also tried to use as a gain medium. Under identical optical pumping conditions, we found no evidence for lasing, most likely the result of scattering-induced optical losses associated with DPFO8 phase separation and crystallization during film formation.

In summary, a novel supramolecular system comprising the newly synthesized DPFOH as a dopant in PMMA was investigated. In stark contrast to DPFO8 system, DPFOH CSP and DPFOH/PMMA SDP systems exhibit an excellent filmforming ability via solution spin-coating process for the formation of hydrogen-bonding interaction. Meanwhile, the hydrogen-bonding interaction between DPFOH and PMMA chain allow for an efficient dispersion of DPFOH molecules in PMMA matrix with a single molecular photo-physical property and relatively high $\Phi_{\mathrm{PLQY}}$ of $\sim 38( \pm 5) \%$. Further evidence for the optical quality of the SDP films was obtained via the demonstration of ultraviolet whispering gallery mode micro ring laser action, peaked at $385 \mathrm{~nm}$. Supramolecular approach is thus shown to be a promising route for low cost optoelectronic devices; allowing construction of high quality homogeneous thin film structures based on solution processes.

\section{ASSOCIATED CONTENT}

Supporting Information. Experimental conditions and methods, materials characteristics, NMR, DSC, TGA, optical analysis, SEM data and additional data. This material is available free of charge via the Internet at http://pubs.acs.org.

\section{AUTHOR INFORMATION}

\section{Corresponding Author}

*E-mail: wei-huang@njtech.edu.cn, iamlhxie@njupt.edu.cn, xcxseu@seu.edu.cn, donal.bradley@mpls.ox.ac.uk

\section{ACKNOWLEDGMENT}

The project was supported by the National Key Basic Research Program of China (973) (2015CB932200), National Natural Science Funds for Excellent Young Scholars (21322402), National Natural Science Foundation of China (21504041, 21502091, 21274064), Doctoral Fund of Ministry of Education of China (20133223110007), Excellent science and technology innovation team of Jiangsu Higher Education Institutions (2013), Priority Academic Program Development of Jiangsu Higher Education Institutions (PAPD YX03001), Natural Science Funds of the Education Committee of Jiangsu Province (15KJB430019), China Postdoctoral Science Foundation (2015M580419), Jiangsu Planned Projects for Postdoctoral Research Funds (1501019B), Open Project from State Key Laboratory of Supramolecular Structure and Materials at Jilin University (sklssm201612), SICAM Fellowship, the University of Oxford and Imperial College London.

\section{REFERENCES}

(1) Xie, L.-H.; Yin, C.-R.; Lai, W.-Y.; Fan, Q.-L.; Huang, W. Prog. Polym. Sci. 2012, 37, 1192.

(2) Yap, B. K.; Xia, R.; Campoy-Quiles, M.; Stavrinou, P. N.; Bradley, D. D. Nature Mater., 2008, 7, 376.

(3) Perevedentsev, A.; Sonnefraud, Y.; Belton, C. R.; Sharma, S.; Cass, A. E.; Maier, S. A.; Kim, J.-S.; Stavrinou, P. N.; Bradley, D. D. Nature Commun., 2015, 6, 5799.

(4) Campoy-Quiles, M.; Ferenczi, T.; Agostinelli, T.; Etchegoin, P. G.; Kim, Y.; Anthopoulos, T. D.; Stavrinou, P. N.; Bradley, D. D.; Nelson, J. Nature Mater., 2008, 7, 158.

(5) Brunsveld, L.; Folmer, B.; Meijer, E.; Sijbesma, R. Chem. Rev., 2001, 101, 4071.

(6) Lehn, J.-M. Chem. Soc. Rev.,2007, 36, 151.

(7) Hoeben, F. J.; Jonkheijm, P.; Meijer, E.; Schenning, A. P. Chem. Rev., 2005, 105, 1491.

(8) Bassani, D. M.; Jonusauskaite, L.; Lavie-Cambot, A.; McClenaghan, N. D.; Pozzo, J.-L.; Ray, D.; Vives, G. Coord. Chem. Rev., 2010, 254, 2429.

(9) Lin, J.; Yu, Z.; Zhu, W.; Xing, G.; Lin, Z.; Yang, S.; Xie, L.; Niu, C.; Huang, W. Polym. Chem., 2013, 4, 477.

(10) Lin, J.-Y.; Zhu, W.-S.; Liu, F.; Xie, L.-H.; Zhang, L.; Xia, R.; Xing, G.-C.; Huang, W. Macromolecules 2014, 47, 1001.

(11) Lin, J. Y.; Wong, J.; Xie, L. H.; Dong, X. C.; Yang, H. Y.; Huang, W. Macromol. Rapid Commun., 2014, 35, 895.

(12) Xie, L.-H.; Ling, Q.-D.; Hou, X.-Y.; Huang, W. J. Am. Chem. Soc., 2008, 130, 2120.

(13) Ji, X.; Yao, Y.; Li, J.; Ytan, X.; Huang, F. J. Am. Chem. Soc., 2013, 135, 74.

(14) Zalar, P.; Henson, Z. B.; Welch, G. C.; Bazan, G. C.; Nguyen, T. Q. Angew. Chem. Int. Ed., 2012, 51, 7495.

(15) Evans, R. C. J. Mater. Chem. C 2013, 1, 4190.

(16) Dudek, S. P.; Pouderoijen, M.; Abbel, R.; Schenning, A. P. H. J.; Meijer, E. W. J. Am. Chem. Soc., 2005, 127, 11763.

(17) Chiu, Y. C.; Otsuka, I.; Halila, S.; Borsali, R.; Chen, W. C. Adv. Funct. Mater., 2014, 24, 4240.

(18) Kuriki, K.; Koike, Y.; Okamoto, Y. Chem. Rev., 2002, 102, 2347.

(19) Ishigure, T.; Nihei, E.; Koike, Y. Appl. Opt., 1994, 33, 4261.

(20) Morello, G.; Camposeo, A.; Moffa, M.; Pisignano, D. ACS Appl. Mater. \& Inter., 2015, 7, 5213.

(21) Lee, O. P.; Yiu, A. T.; Beaujuge, P. M.; Woo, C. H.; Holcombe, T. W.; Millstone, J. E.; Douglas, J. D.; Chen, M. S.; Fréchet, J. M. Adv., Mater., 2011, 23, 5359.

(22) Liao, M. H.; Tsai, C. E.; Lai, Y. Y.; Cao, F. Y.; Wu, J. S.; Wang, C. L.; Hsu, C. S.; Liau, I.; Cheng, Y. J. Adv. Funct. Mater., 2014, 24, 1418.

(23) Zhang, G.-W.; Wang, L.; Xie, L.-H.; Lin, J.-Y.; Huang, W. Int. J. Mol. Sci., 2013, 14, 22368.

(24) Sims, M.; Bradley, D. D.; Ariu, M.; Koeberg, M.; Asimakis, A.; Grell, M.; Lidzey, D. G. Adv. Funct. Mater., 2004, 14, 765.

(25) Chan, K. L.; Sims, M.; Pascu, S. I.; Ariu, M.; Holmes, A. B.; Bradley, D. D. Adv. Funct. Mater., 2009, 19, 2147.

(26) Mo, Y.; Du, L.; Liu, L.; Huang, J.; Pan, Y.; Yang, B.; Xie, Z.; Ma, Y. J. Phys. Chem. C 2013, 117, 27081.

(27) Kobayashi, T.; Byrne, N. Appl. Phys. Lett., 2011, 99, 153307.

(28) Zhu, G.; Xu, C.; Cai, L.; Li, J.; Shi, Z.; Lin, Y.; Chen, G.; Ding, T.; Tian, Z.; Dai, J. ACS Appl. Mater.\& Inter., 2012, 4, 6195.

Table of Contents 


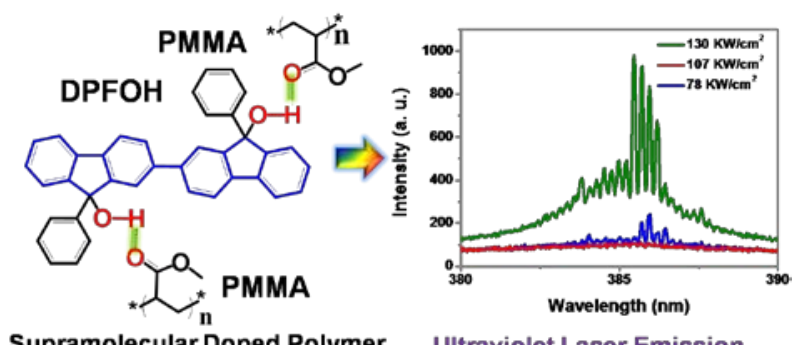

Supramolecular Doped Polymer Ultraviolet Laser Emission 